Research Article

\title{
Two Classes of Topological Indices of Phenylene Molecule Graphs
}

\author{
Tingzeng Wu \\ School of Mathematics and Statistics, Qinghai Nationalities University, Xining, Qinghai 810007, China \\ Correspondence should be addressed to Tingzeng Wu; mathtzwu@163.com
}

Received 1 March 2016; Accepted 3 April 2016

Academic Editor: Hua Fan

Copyright (C) 2016 Tingzeng Wu. This is an open access article distributed under the Creative Commons Attribution License, which permits unrestricted use, distribution, and reproduction in any medium, provided the original work is properly cited.

A phenylene is a conjugated hydrocarbons molecule composed of six- and four-membered rings. The matching energy of a graph $G$ is equal to the sum of the absolute values of the zeros of the matching polynomial of $G$, while the Hosoya index is defined as the total number of the independent edge sets of $G$. In this paper, we determine the extremal graph with respect to the matching energy and Hosoya index for all phenylene chains.

\section{Introduction}

Phenylenes are a class of conjugated hydrocarbons composed of six- and four-membered rings, where the six-membered rings (hexagons) are adjacent only to four-membered rings, and every four-membered ring is adjacent to a pair of hexagons. They are nanostructures that can be precisely designed and manufactured for a wide variety of applications; see [1-3] and the references therein.

A topological index is a numerical quantity derived in an unambiguous manner from the structure graph of a molecule, as a graph structural invariant; that is, it does not depend on the labeling or the pictorial representation of a graph. Various topological indices usually reflect molecular size and shape. One topological index is Hosoya index, which was first introduced by Hosoya [4]. It plays an important role in the so-called inverse structure-property relationship problems. For details of Hosoya index and its applications, the readers are suggested to refer to $[5,6]$. A new topological index in chemistry, matching energy, is first introduced by Gutman and Wagner [7] in 2012 to study topological resonance energy of conjugated molecules, which has received a lot of attention from researchers in recent years. For more background and applications about matching energy, see [8-16].

In this paper, our aim is to determine the phenylenes with minimum and maximum matching energy (Hosoya index) among all the phenylenes with $n$ hexagons.
In the following we present some definitions and notations.

Let $G=(V, E)$ be a graph with the vertex set $V(G)$ and the edge set $E(G)$. Let $e$ and $v$ be an edge and a vertex in $G$, respectively. We denote by $G-e$ the graph obtained from $G$ by removing edge $e$ and by $G-v$ the graph obtained from $G$ by deleting vertex $v$.

By $m(G, k)$ we denote the number of $k$-matchings of a graph $G$. The matching polynomial of a graph $G$ with $n$ vertices is fined as

$$
\alpha(G, x)=\sum_{k \geq 0}(-1)^{k} m(G, k) x^{n-2 k}
$$

where $m(G, 0)=1$ and $m(G, k) \geq 0$ for all $k=1,2, \ldots,[n / 2]$. This expression $\alpha(G, x)$ induces a quasi-order relation (i.e., reflexive and transitive relation) on the set of all graphs with $n$ vertices. If $G$ and $H$ are two graphs with matching polynomial in the form (1), then the quasi-order $\succeq$ is defined by

$$
\begin{aligned}
& G \geq H \Longleftrightarrow m(G, k) \geq m(H, k) \\
& \forall k=0,1, \ldots,\lfloor n / 2\rfloor .
\end{aligned}
$$

Particularly, if $G \geq H$ and there exists some $k$ such that $m(G, k)>m(H, k)$, then we write $G>H$. 
Gutman and Wagner in [7] first proposed the concept of the matching energy of a graph, denoted by $\operatorname{ME}(G)$, as

$$
\mathrm{ME}=\operatorname{ME}(G)=\frac{2}{\pi} \int_{0}^{\infty} x^{-2} \ln \left[\sum_{k \geq 0} m(G, k) x^{2 k}\right] d x .
$$

Meanwhile, they gave also another form of definition of matching energy of a graph. That is,

$$
\operatorname{ME}(G)=\sum_{i=1}^{n}\left|\mu_{i}\right|,
$$

where $\mu_{i}$ denotes the root of matching polynomial of $G$. By (2) and (3), we easily obtain the fact as follows:

$$
\begin{aligned}
& G \geq H \Longrightarrow \operatorname{ME}(G) \geq \operatorname{ME}(H), \\
& G>H \Longrightarrow \operatorname{ME}(G)>\operatorname{ME}(H) .
\end{aligned}
$$

The $Z$-counting polynomial was defined by Hosoya [4] as

$$
Z(G)=Z(G, x)=\sum_{k} m(G, k) x^{k} .
$$

Particularly, set $x=1$; then $Z(G, 1)=\sum_{k} m(G, k)$ is called Hosoya index of $G$. Furthermore, The $Z$-counting polynomial of graphs has the property as follows.

Lemma 1 (see [4]). (a) Let $G$ be a graph consisting of two components $G_{1}$ and $G_{2}$. Then $Z(G)=Z\left(G_{1}\right) Z\left(G_{2}\right)$.

(b) Let $u v \in E(G)$ be an edge of $G$. Then $Z(G)=Z(G-$ $u v)+x Z(G-u-v)$.

A phenylene chain containing $n(n \geq 2)$ hexagons, denoted by $\mathrm{PHB}_{n}$, is a phenylene with the properties that (a) no vertex is incident with two hexagons or squares and (b) no hexagon is adjacent to more than two squares. We denote by $\mathscr{B}_{n}$ the set of all phenylene chains with $n$ hexagons. Let $\mathrm{PHB}_{n} \in \mathscr{B}_{n}$. If the subgraph $\mathrm{PHB}_{n}$ induced by the vertices with degree 3 is the union of $n-1$ disjoint copies of a square, then $\mathrm{PHB}_{n}$ is called a linear phenylene chain and denoted by $\mathrm{PHL}_{n}$ (see Figure 1). If the subgraph $\mathrm{PHB}_{n}$ of induced by the vertices with degree 3 is isomorphic to the graph $S_{n-1}$ having $n-1$ squares (see Figure 1), then $\mathrm{PHB}_{n}$ is called a zigzag phenylene chain and is denoted by $\mathrm{PHZ}_{n}$ (see Figure 1). It is easy to see that $\mathscr{B}_{2}=\left\{\mathrm{PHL}_{2}\right\}=\left\{\mathrm{PHZ}_{2}\right\}$ and $\mathscr{B}_{3}=\left\{\mathrm{PHL}_{3}, \mathrm{PHZ}_{3}\right\}$. Finally, by the definition of a phenylene chain, any element $\mathrm{PHB}_{n}$ in $\mathscr{B}_{n}$ can be obtained from an appropriately chosen graph $\mathrm{PHB}_{n-1} \in \mathscr{B}_{n-1}$ by attaching to it a new graph $\theta$, where $\theta$ is obtained from an edge of a square attaching an edge of a hexagon; see Figure 2.

\section{Main Results}

Theorem 2. Let $\mathscr{B}_{n}$ be the set of all phenylene chains with $n$ hexagons. For any $\mathrm{PHB}_{n} \in \mathscr{B}_{n}$, then

$$
\operatorname{ME}\left(P H L_{n}\right) \leq M E\left(P H B_{n}\right) \leq M E\left(P H Z_{n}\right),
$$

where the equalities on the left side hold only if $P H B_{n} \cong P H L_{n}$, and the equalities on the right side hold only if $P H B_{n} \cong P H Z_{n}$.

By (2) and (5), we know that Theorem 2 holding only needs to prove the following result.

Theorem 3. For any $\mathrm{PHB}_{n} \in \mathscr{B}_{n}$ and for each $k \geq 0$,

$$
m\left(P H L_{n}, k\right) \leq m\left(P H B_{n}, k\right) \leq m\left(P H Z_{n}, k\right),
$$

where the equalities on the left side hold only if $P H B_{n} \cong P H L_{n}$ and the equalities on the right side hold only if $P H B_{n} \cong P H Z_{n}$.

Let $f(x)=\sum_{k=0}^{n} a_{k} x^{k}$ and $g(x)=\sum_{k=0}^{n} b_{k} x^{k}$ be two polynomials of $x$. We say $f(x) \preceq g(x)$ if $a_{k} \leq b_{k}$ for all $k$. If $f(x) \preceq g(x)$ and there exists some $k$ such that $a_{k}<b_{k}$, then we say $f(x) \prec g(x)$. By (6), it is easy to obtain the following result which is equivalent to Theorem 3 .

Theorem 4. For any $\mathrm{PHB}_{n} \in \mathscr{B}_{n}(n \geq 2)$,

(I) if $P H L_{n} \neq P H B_{n}$, then $Z\left(P H L_{n}\right) \prec Z\left(P H B_{n}\right)$,

(II) if $P H Z_{n} \neq P H B_{n}$, then $Z\left(P H B_{n}\right) \prec Z\left(P H Z_{n}\right)$.

In the following we will use the notation $G$ for $Z(G)$, when it would lead to no confusion.

Proof. Checking Figure 2, by Lemma 1, we obtained that

$$
\begin{aligned}
& \mathrm{PHB}_{n}=\left(1+6 x+9 x^{2}+2 x^{3}\right) \mathrm{PHB}_{n-1}+\left(x+4 x^{2}+3 x^{3}\right)\left[\left(\mathrm{PHB}_{n-1}-s_{2 n-3}\right)+\left(\mathrm{PHB}_{n-1}-t_{2 n-3}\right)\right]+\left(x^{2}+3 x^{3}+x^{4}\right)\left(\mathrm{PHB}_{n-1}-s_{2 n-3}-t_{2 n-3}\right) \text {, } \\
& =\left\{\begin{array}{l}
\left(1+4 x+3 x^{2}\right) \mathrm{PHB}_{n-1}+\left(x+2 x^{2}\right)\left(\mathrm{PHB}_{n-1}-t_{2 n-3}\right)+\left(x+3 x^{2}+x^{3}\right)\left(\mathrm{PHB}_{n-1}-s_{2 n-3}\right)+\left(x^{2}+2 x^{3}\right)\left(\mathrm{PHB}_{n-1}-s_{2 n-3}-t_{2 n-3}\right) \quad \text { if } y=b_{1}^{n}, \\
\left(1+4 x+3 x^{2}\right) \mathrm{PHB}_{n-1}+\left(x+2 x^{2}+x^{3}\right)\left(\mathrm{PHB}_{n-1}-t_{2 n-3}\right)+\left(x+2 x^{2}\right)\left(\mathrm{PHB}_{n-1}-s_{2 n-3}\right)+\left(x^{2}+x^{3}\right)\left(\mathrm{PHB}_{n-1}-s_{2 n-3}-t_{2 n-3}\right) \quad \text { if } y=b_{2}^{n}, \\
\left(1+4 x+3 x^{2}\right) \mathrm{PHB}_{n-1}+\left(x+2 x^{2}+x^{3}\right)\left(\mathrm{PHB}_{n-1}-s_{2 n-3}\right)+\left(x+2 x^{2}\right)\left(\mathrm{PHB}_{n-1}-t_{2 n-3}\right)+\left(x^{2}+x^{3}\right)\left(\mathrm{PHB}_{n-1}-s_{2 n-3}-t_{2 n-3}\right) \quad \text { if } y=b_{3}^{n}, \\
\left(1+4 x+3 x^{2}\right) \mathrm{PHB}_{n-1}+\left(x+2 x^{2}\right)\left(\mathrm{PHB}_{n-1}-s_{2 n-3}\right)+\left(x+3 x^{2}+x^{3}\right)\left(\mathrm{PHB}_{n-1}-t_{2 n-3}\right)+\left(x^{2}+2 x^{3}\right)\left(\mathrm{PHB}_{n-1}-s_{2 n-3}-t_{2 n-3}\right) \quad \text { if } y=b_{4}^{n},
\end{array}\right.
\end{aligned}
$$

$\mathrm{PHB}_{n}-y-z$

$$
= \begin{cases}\left(1+3 x+x^{2}\right) \mathrm{PHB}_{n-1}+\left(x+x^{2}\right)\left(\mathrm{PHB}_{n-1}-t_{2 n-3}\right)+\left(x+2 x^{2}\right)\left(\mathrm{PHB}_{n-1}-s_{2 n-3}\right)+\left(x^{2}+x^{3}\right)\left(\mathrm{PHB}_{n-1}-s_{2 n-3}-t_{2 n-3}\right) & \text { if } y=b_{1}^{n}, z=b_{2}^{n} ; \\ \left(1+3 x+x^{2}\right) \mathrm{PHB}_{n-1}+\left(x+x^{2}\right)\left(\mathrm{PHB}_{n-1}-t_{2 n-3}\right)+\left(x+x^{2}\right)\left(\mathrm{PHB}_{n-1}-s_{2 n-3}\right)+x\left(\mathrm{PHB}_{n-1}-s_{2 n-3}-t_{2 n-3}\right) & \text { if } y=b_{2}^{n}, z=b_{3}^{n} ; \\ \left(1+3 x+x^{2}\right) \mathrm{PHB}_{n-1}+\left(x+x^{2}\right)\left(\mathrm{PHB}_{n-1}-s_{2 n-3}\right)+\left(x+2 x^{2}\right)\left(\mathrm{PHB}_{n-1}-t_{2 n-3}\right)+\left(x^{2}+x^{3}\right)\left(\mathrm{PHB}_{n-1}-s_{2 n-3}-t_{2 n-3}\right) & \text { if } y=b_{3}^{n}, z=b_{4}^{n} .\end{cases}
$$



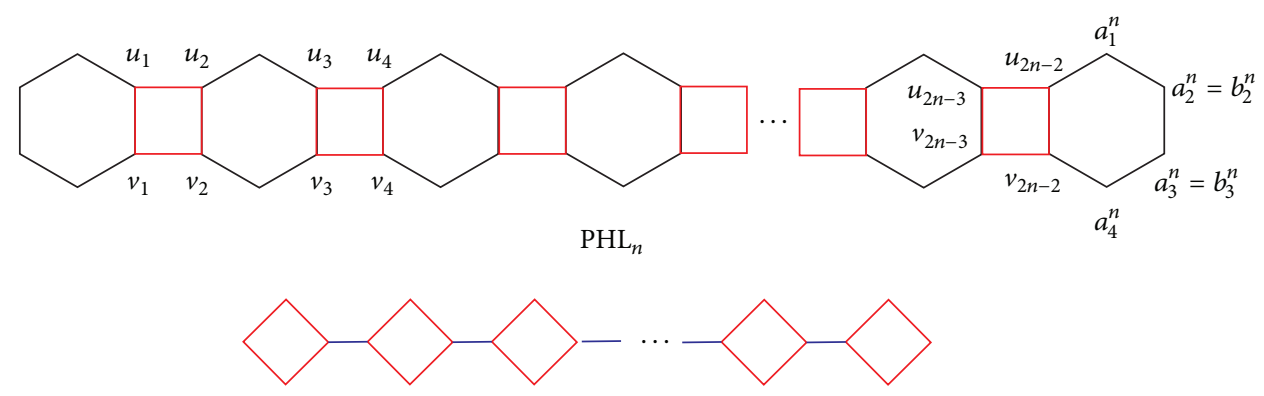

$S_{n-1}$

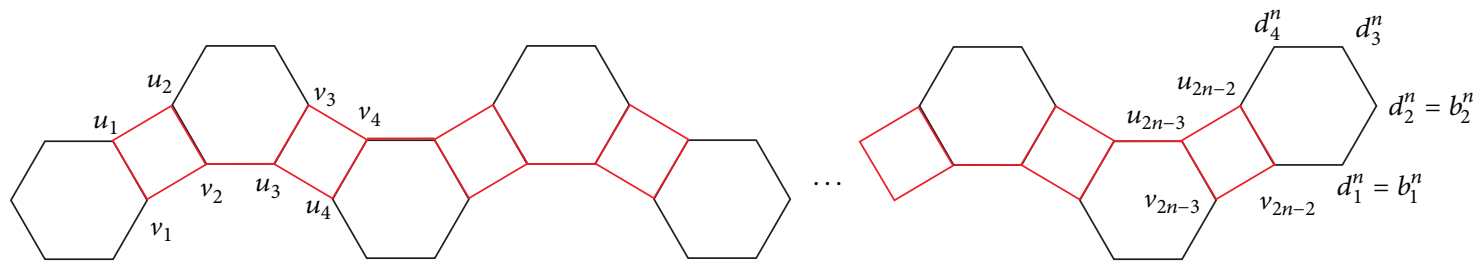

$\mathrm{PHZ}_{n}$

FIgURE 1: A linear phenylene chain $\mathrm{PHL}_{n}$ and a zigzag phenylene chain $\mathrm{PHZ}_{n}$.

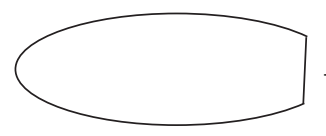

$\mathrm{PHB}_{n-1}$

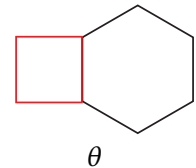

Figure 2: A phenylene chain $\mathrm{PHB}_{n}$
By (10) and (11), we have

(i) $\mathrm{PHB}_{n}-b_{3}^{n} \prec \mathrm{PHB}_{n}-b_{1}^{n}$ and $\mathrm{PHB}_{n}-b_{2}^{n} \prec \mathrm{PHB}_{n}-b_{4}^{n}$;

(ii) $\mathrm{PHB}_{n}-b_{2}^{n}-b_{3}^{n} \prec \mathrm{PHB}_{n}-b_{1}^{n}-b_{2}^{n}$ and $\mathrm{PHB}_{n}-b_{2}^{n}-b_{3}^{n} \prec$ $\mathrm{PHB}_{n}-b_{3}^{n}-b_{4}^{n}$.

Particularly, if $\mathrm{PHB}_{n}=\mathrm{PHL}_{n}$, then

(i') $\mathrm{PHL}_{n}-a_{2}^{n}=\mathrm{PHL}_{n}-a_{3}^{n} \prec \mathrm{PHL}_{n}-a_{1}^{n}=\mathrm{PHL}_{n}-a_{4}^{n}$,

(ii') $\mathrm{PHL}_{n}-a_{2}^{n}-a_{3}^{n} \prec \mathrm{PHL}_{n}-a_{1}^{n}-a_{2}^{n}=\mathrm{PHL}_{n}-a_{3}^{n}-a_{4}^{n}$,

$\left(\right.$ iii' $\left.^{\prime}\right)\left(\mathrm{PHL}_{n}-a_{2}^{n}\right)+\left(\mathrm{PHL}_{n}-a_{3}^{n}\right) \prec\left(\mathrm{PHL}_{n}-a_{2}^{n}\right)+\left(\mathrm{PHL}_{n}-\right.$ $\left.a_{1}^{n}\right)=\left(\mathrm{PHL}_{n}-a_{3}^{n}\right)+\left(\mathrm{PHL}_{n}-a_{4}^{n}\right)$.

We prove Theorem 4(I) by mathematical induction.

First we consider $n=3$. In this case, $\mathscr{B}_{n}=\left\{\mathrm{PHL}_{3}, \mathrm{PHZ}_{3}\right\}$. By (9), we have

$\mathrm{PHL}_{3}$

$$
\begin{aligned}
= & \left(1+6 x+9 x^{2}+2 x^{3}\right) \mathrm{PHL}_{2} \\
& +\left(x+4 x^{2}+3 x^{3}\right)\left[\left(\mathrm{PHL}_{2}-a_{2}^{2}\right)+\left(\mathrm{PHL}_{2}-a_{3}^{2}\right)\right] \\
& +\left(x^{2}+3 x^{3}+x^{4}\right)\left(\mathrm{PHL}_{2}-a_{2}^{2}-a_{3}^{2}\right),
\end{aligned}
$$

$\mathrm{PHZ}_{3}$

$$
\begin{aligned}
= & \left(1+6 x+9 x^{2}+2 x^{3}\right) \mathrm{PHZ}_{2} \\
& +\left(x+4 x^{2}+3 x^{3}\right)\left[\left(\mathrm{PHZ}_{2}-d_{1}^{2}\right)+\left(\mathrm{PHZ}_{2}-d_{2}^{2}\right)\right] \\
& +\left(x^{2}+3 x^{3}+x^{4}\right)\left(\mathrm{PHZ}_{2}-d_{1}^{2}-d_{2}^{2}\right) \\
= & \left(1+6 x+9 x^{2}+2 x^{3}\right) \mathrm{PHL}_{2} \\
& +\left(x+4 x^{2}+3 x^{3}\right)\left[\left(\mathrm{PHL}_{2}-a_{4}^{2}\right)+\left(\mathrm{PHL}_{2}-a_{3}^{2}\right)\right] \\
& +\left(x^{2}+3 x^{3}+x^{4}\right)\left(\mathrm{PHL}_{2}-a_{3}^{2}-a_{4}^{2}\right) .
\end{aligned}
$$

By $\left(\mathrm{i}^{\prime}\right)-\left(\mathrm{iii}^{\prime}\right)$, we have $\mathrm{PHL}_{3} \prec \mathrm{PHZ}_{3}$.

Suppose that Theorem $4(\mathrm{I})$ is right for all phenylene chains with few $n$ hexagons. Let $\mathrm{PHB}_{n}$ be a phenylene chain with $n \geq 4$ hexagons, which is obtained from $\mathrm{PHB}_{n-1} \in \mathscr{B}_{n-1}$ by attaching to it a new $\theta$ (see Figure 2). We show that if $\mathrm{PHL}_{n} \neq \mathrm{PHB}_{n}$, then $\mathrm{PHL}_{n} \prec \mathrm{PHB}_{n}$. By (9) we obtain that

$$
\begin{aligned}
& \mathrm{PHL}_{n}=\left(1+6 x+9 x^{2}+2 x^{3}\right) \mathrm{PHL}_{n-1} \\
& +\left(x+4 x^{2}+3 x^{3}\right)
\end{aligned}
$$




$$
\begin{aligned}
& \cdot\left[\left(\mathrm{PHL}_{n-1}-u_{2 n-3}\right)+\left(\mathrm{PHL}_{n-1}-v_{2 n-3}\right)\right] \\
& +\left(x^{2}+3 x^{3}+x^{4}\right)\left(\mathrm{PHL}_{n-1}-u_{2 n-3}-v_{2 n-3}\right), \\
& \mathrm{PHB}_{n}=\left(1+6 x+9 x^{2}+2 x^{3}\right) \mathrm{PHB}_{n-1} \\
& +\left(x+4 x^{2}+3 x^{3}\right) \\
& \cdot\left[\left(\mathrm{PHB}_{n-1}-s_{2 n-3}\right)+\left(\mathrm{PHB}_{n-1}-t_{2 n-3}\right)\right] \\
& +\left(x^{2}+3 x^{3}+x^{4}\right)\left(\mathrm{PHB}_{n-1}-s_{2 n-3}-t_{2 n-3}\right) .
\end{aligned}
$$

By the inductive hypotheses we have $\mathrm{PHL}_{n-1} \preceq \mathrm{PHB}_{n-1}$, $\left(\mathrm{PHL}_{n-1}-u_{2 n-3}\right)+\left(\mathrm{PHL}_{n-1}-v_{2 n-3}\right) \preceq\left(\mathrm{PHB}_{n-1}-s_{2 n-3}\right)+$ $\left(\mathrm{PHB}_{n-1}-t_{2 n-3}\right)$, and $\left(\mathrm{PHL}_{n-1}-u_{2 n-3}-v_{2 n-3}\right) \preceq\left(\mathrm{PHB}_{n-1}-\right.$ $\left.s_{2 n-3}-t_{2 n-3}\right)$. Since $\mathrm{PHL}_{n} \neq \mathrm{PHB}_{n}$, either $\mathrm{PHL}_{n-1} \neq \mathrm{PHB}_{n-1}$ or $\left\{s_{n-1}, t_{n-1}\right\} \neq\left\{u_{2 n-3}, v_{2 n-3}\right\}$, and hence at least one of the three inequalities is strict. Therefore, we get that $\mathrm{PHL}_{n} \prec$ $\mathrm{PHB}_{n}$.

In the following we prove Theorem 4(II) by induction.

By the proof of Theorem 4(I), we know that $\mathrm{PHB}_{3} \prec$ $\mathrm{PHZ}_{3}$.

Similarly, suppose that Theorem 4(II) is right for all phenylene chains with few $n$ hexagons. Let $\mathrm{PHB}_{n}$ be a phenylene chain with $n \geq 4$ hexagons, which is obtained from $\mathrm{PHB}_{n-1} \in \mathscr{B}_{n-1}$ by attaching to it a new $\theta$ (see Figure 2 ). We show that if $\mathrm{PHB}_{n} \neq \mathrm{PHZ}_{n}$, then $\mathrm{PHB}_{n} \prec \mathrm{PHZ}_{n}$. By (9) we have

$$
\begin{aligned}
& \mathrm{PHB}_{n}=\left(1+6 x+9 x^{2}+2 x^{3}\right) \mathrm{PHB}_{n-1} \\
& +\left(x+4 x^{2}+3 x^{3}\right) \\
& \cdot\left[\left(\mathrm{PHB}_{n-1}-s_{2 n-3}\right)+\left(\mathrm{PHB}_{n-1}-t_{2 n-3}\right)\right] \\
& \quad+\left(x^{2}+3 x^{3}+x^{4}\right)\left(\mathrm{PHB}_{n-1}-s_{2 n-3}-t_{2 n-3}\right) \\
& \mathrm{PHZ}_{n}=\left(1+6 x+9 x^{2}+2 x^{3}\right) \mathrm{PHZ}_{n-1} \\
& +\left(x+4 x^{2}+3 x^{3}\right) \\
& \quad \cdot\left[\left(\mathrm{PHZ}_{n-1}-u_{2 n-3}\right)+\left(\mathrm{PHZ}_{n-1}-v_{2 n-3}\right)\right] \\
& +\left(x^{2}+3 x^{3}+x^{4}\right)\left(\mathrm{PHZ}_{n-1}-u_{2 n-3}-v_{2 n-3}\right) .
\end{aligned}
$$

By the inductive hypotheses we have $\mathrm{PHB}_{n-1} \preceq \mathrm{PHZ}_{n-1}$, $\left(\mathrm{PHB}_{n-1}-s_{2 n-3}\right)+\left(\mathrm{PHB}_{n-1}-t_{2 n-3}\right) \preceq\left(\mathrm{PHZ}_{n-1}-u_{2 n-3}\right)+$ $\left(\mathrm{PHZ}_{n-1}-v_{2 n-3}\right)$, and $\left(\mathrm{PHB}_{n-1}-s_{2 n-3}-t_{2 n-3}\right) \preceq\left(\mathrm{PHZ}_{n-1}-\right.$ $\left.u_{2 n-3}-v_{2 n-3}\right)$. Since $\mathrm{PHB}_{n} \neq \mathrm{PHZ}_{n}$, either $\mathrm{PHB}_{n-1} \neq \mathrm{PHZ}_{n-1}$ or $\left\{s_{n-1}, t_{n-1}\right\} \neq\left\{u_{2 n-3}, v_{2 n-3}\right\}$, and hence at least one of the three inequalities is strict. Therefore, we get that $\mathrm{PHB}_{n} \prec$ $\mathrm{PHZ}_{n}$.

The proof is complete.

By the definition of Hosoya index and Theorem 4, we can obtain the following result.

Theorem 5. Let $\mathscr{B}_{n}$ be the set of all phenylene chains with $n$ hexagons. For any $\mathrm{PHB}_{n} \in \mathscr{B}_{n}$, then

$$
Z\left(P H L_{n}, 1\right) \leq Z\left(P H B_{n}, 1\right) \leq Z\left(P H Z_{n}, 1\right),
$$

where the equalities on the left side hold only if $P H B_{n} \cong P H L_{n}$ and the equalities on the right side hold only if $P H B_{n} \cong P H Z_{n}$.

\section{Competing Interests}

The author declares that there are no competing interests regarding the publication of this paper.

\section{Acknowledgments}

This work is financially supported by the National Natural Science Foundation of China (11371180 and 11561056), the Project of QHMU (2015XJZ12), and the Qinghai Province Natural Science Foundation (2016).

\section{References}

[1] C. Dosche, H. Löhmannsröben, A. Bieser et al., "Photophysical properties of $[N]$ phenylenes," The Journal of Physical Chemistry $A$, vol. 102, no. 41, pp. 8051-8055, 1998.

[2] J. Rada, O. Araujo, and I. Gutman, "Randić index of benzenoid systems and phenylenes," Croatica Chemica Acta, vol. 74, no. 2, pp. 225-235, 2001.

[3] J. M. Schulman, R. L. Disch, H. Jiao, and P. Von Ragué Schleyer, "Chemical shifts of the $[N]$ phenylenes and related compounds," Journal of Physical Chemistry A, vol. 102, no. 41, pp. 8051-8055, 1998.

[4] H. Hosoya, "Topological index," The Bulletin of the Chemical Society of Japan, vol. 44, no. 9, pp. 2332-2339, 1971.

[5] S. Wagner and I. Gutman, "Maxima and minima of the Hosoya index and the Merrifield-Simmons index: a survey of results and techniques," Acta Applicandae Mathematicae, vol. 112, no. 3, pp. 323-346, 2010.

[6] K. Xu, "Computing the hosoya index and the wiener index of an infinite class of dendrimers," Digest Journal of Nanomaterials and Biostructures, vol. 6, pp. 265-270, 2011.

[7] I. Gutman and S. Wagner, "The matching energy of a graph," Discrete Applied Mathematics, vol. 160, no. 15, pp. 2177-2187, 2012.

[8] L. Chen, J. Liu, and Y. Shi, "Matching energy of unicyclic and bicyclic graphs with a given diameter," Complexity, vol. 21, no. 2, pp. 224-238, 2015.

[9] L. Chen and Y. Shi, "The maximal matching energy of tricyclic graphs," MATCH Communications in Mathematical and in Computer Chemistry, vol. 73, pp. 105-119, 2015.

[10] X. Chen, X. Li, and H. Lian, "The matching energy of random graphs," Discrete Applied Mathematics, vol. 193, pp. 102-109, 2015.

[11] L. Feng, "The Laplacian spectral radius of graphs with given matching number," Ars Combinatoria, vol. 96, pp. 257-262, 2010.

[12] S. Ji, X. Li, and Y. Shi, "Extremal matching energy of bicyclic graphs," MATCH Communications in Mathematical and in Computer Chemistry, vol. 70, no. 2, pp. 697-706, 2013.

[13] S. Li and W. Yan, "The matching energy of graphs with given parameters," Discrete Applied Mathematics, vol. 162, pp. 415420, 2014.

[14] T. Wu, W. Yan, and H. Zhang, "Extremal matching energy of complements of trees," Discussiones Mathematicae Graph Theory, 2016. 
[15] K. Xu, K. C. Das, and Z. Zheng, “The minimal matching energy of (n;m)-graphs with a given matching number," MATCH Communications in Mathematical and in Computer Chemistry, vol. 73, pp. 93-104, 2015.

[16] K. Xu, Z. Zheng, and K. C. Das, "Extremal $t$-apex trees with respect to matching energy," Complexity, 2015. 


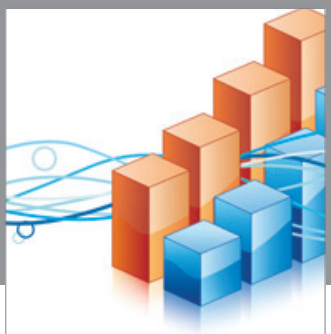

Advances in

Operations Research

vatem alat4

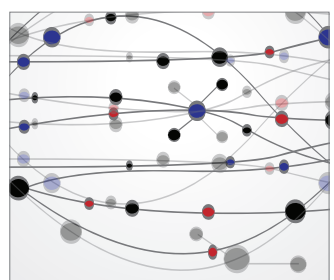

\section{The Scientific} World Journal
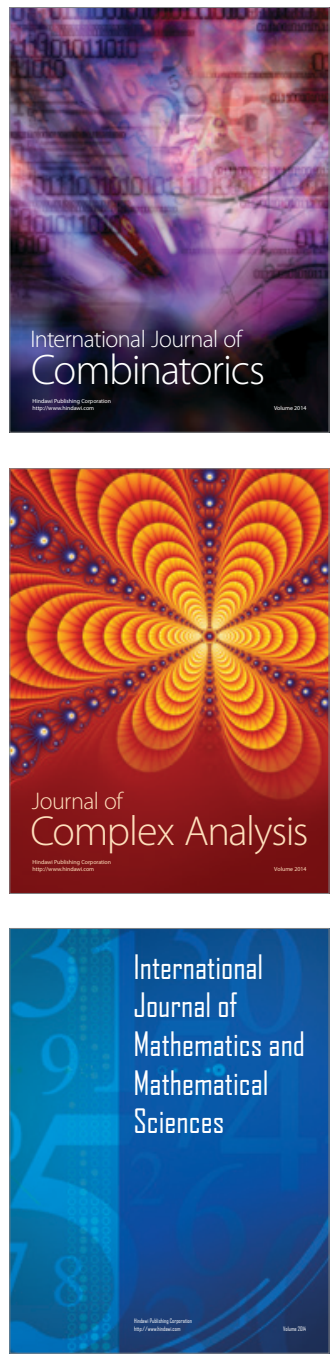
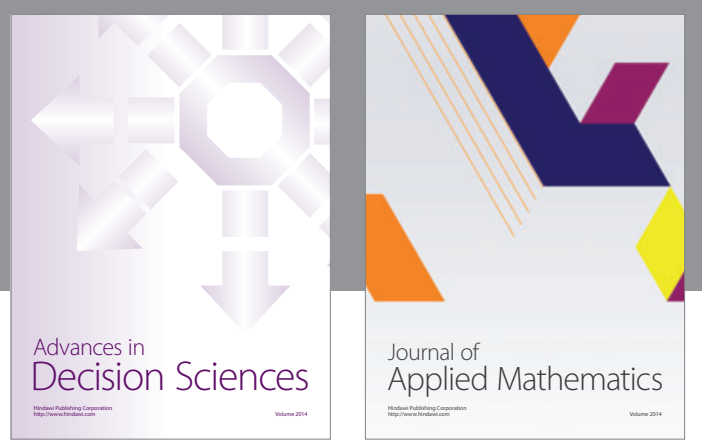

Algebra

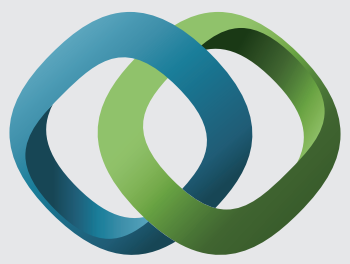

\section{Hindawi}

Submit your manuscripts at

http://www.hindawi.com
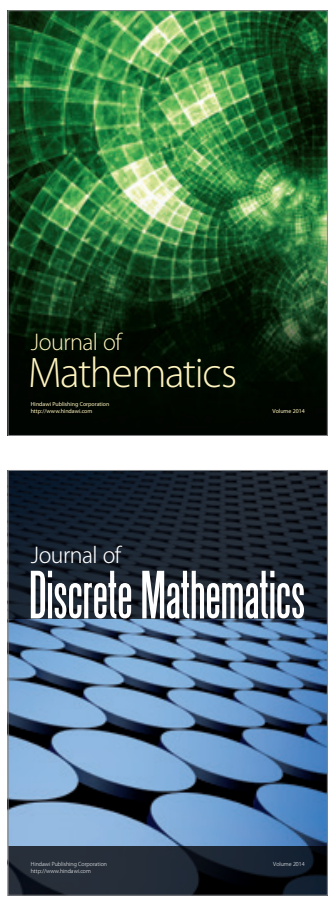

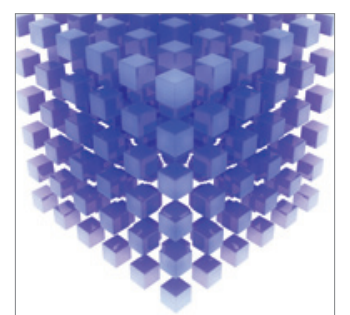

Mathematical Problems in Engineering
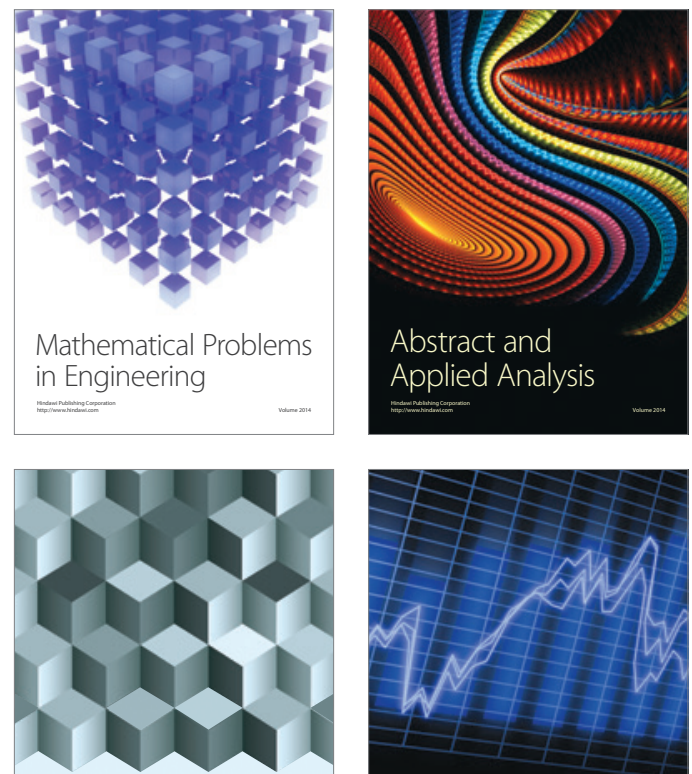

Journal of

Function Spaces

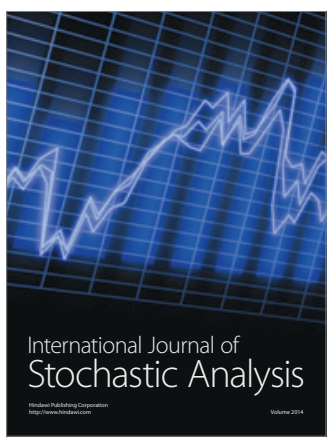

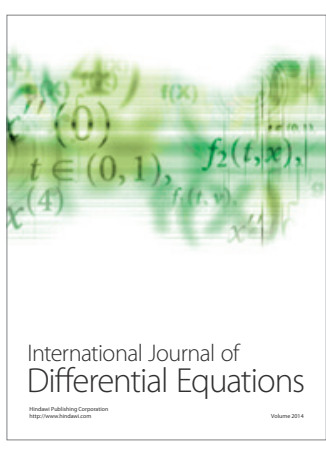
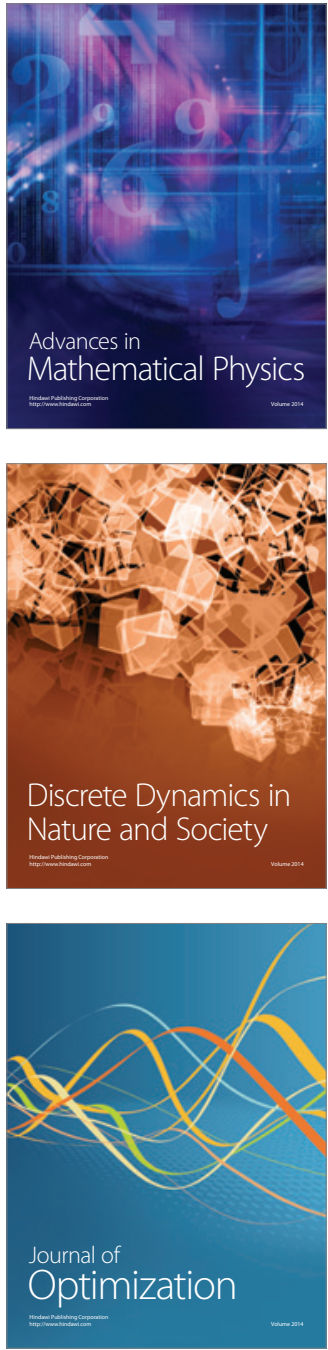